\title{
Diagnostic Accuracy of Axillary Ultrasonography Compared with Intra-operative Pathological Findings in Patients with Breast Cancer
}

\author{
Alireza Rezvani ${ }^{1}$, Aryan Zahergivar ${ }^{2 *}$, Pooya Iranpour ${ }^{3}$, Majid Akrami ${ }^{1}$, Sepehr \\ Kazemi $^{1}$
}

\begin{abstract}
Objective: Breast cancer is the most common cancer and leading cause of mortality in women worldwide. Diagnosis of axillary lymph node involvement, frequently by axillary ultrasonography (AUS), is an important step in patients with primary breast cancer, while the gold standard pathological examination is only applicable during surgery. Thus, we aimed to evaluate the predictive value of AUS in detection of lymph node involvement. Methods: A total of 56 patients with primary breast cancer, who were candidate of mastectomy were included in this study. A single radiologist performed pre-operative axillary sonography for all the patients and reported the results in a checklist. The results were then compared with intra-operative pathological results of lymph node dissection to evaluate the sensitivity, specificity, positive and negative predictive values of AUS in detection of the disease. Result: The results showed that pre-operative AUS had a sensitivity of $63.3 \%$, specificity of $84.6 \%$, positive predictive value of $82.6 \%$, and negative predictive value of $66.6 \%$. Correlation between axillary lymph node characteristics revealed that the absence of fatty hilum, cortical thickness, and loss of ovality of the lymph nodes were the most specific factors in detection of lymph nodes' involvement. Conclusion: AUS has a low sensitivity and cannot determine the presence of micro-metastases and suspicious lymph nodes accurately in early stages of the disease, and it seems that defining new parameters may help for increasing the sensitivity and specificity of the axillary lymph node sonography. Apparently, pathological examination remains the gold standard diagnostic method.
\end{abstract}

Keywords: Breast neoplasms- ultrasonography- lymph nodes- Axilla

Asian Pac J Cancer Prev, 19 (12), 3615-3621

\section{Introduction}

Breast cancer is the most common malignancy in women worldwide (Ghoncheh et al., 2016) with an estimated lifetime chance of 1 in every 8 women in the United States (DeSantis et al., 2014). The lifestyle change and increased life expectancy led to a dramatic increase in the incidence of breast cancer worldwide, while more than half of new cases rise from developing countries (Ngoma, 2006). Furthermore, the peak age of the disease is younger in Asian countries than Western countries (40-50 vs. 60-70 years) (Leong et al., 2010). In Iran, breast cancer accounts for about $25 \%$ of all cancers with an incidence of 22.6, prevalence of 120 (Mousavi et al., 2007), and age-standardized rate of 27.4 per 100,000 women in 2015 (Jazayeri et al., 2015).

The 5-year survival rate depends on the stage of the disease, varying from $99 \%$ in localized stages and $84 \%$ in regional disease to $23 \%$ in metastatic breast cancer (Shah and Guraya, 2017). The preventive measures have substantially increased the survival rate of patients with breast cancer in the United States to about 90\% (Siegel et al., 2018), while it remains at 57\% in developing countries (da Costa Vieira et al., 2017).

Axillary ultrasonography (AUS) has increased the diagnostic accuracy, especially if it is combined with routine mammography (Berg et al., 2008). It is highly suggested for palpable breast mass in young women with dense breasts or breast implants, differentiation between cystic or solid mass, assessment of peripheral masses, and axillary lymph nodes (Pan, 2016). In addition to diagnostic value of axillary nodes' fine needle biopsy under ultrasonography guide (Jung et al., 2010), AUS is suggested as a useful diagnostic tool for patients with breast cancer and palpable mass (Ertan et al., 2013) that can also reduce the number of sentinel node procedures (Deurloo et al., 2003). However, the diagnostic accuracy of ultrasonography differs based on the operator's skills and experience, ultrasound equipment, and examination procedures with limited standardization 
(Ohuchi et al., 2009). Therefore, pathological examination of the breast and axillary lymph nodes during surgery remains the gold standard diagnostic method for tumor, node, metastasis (TNM) staging (Petropoulou et al., 2017).

In the present study, we aimed to determine the diagnostic accuracy of pre-operational AUS in our clinical setting on this population, by comparing the results with the pathological results, obtained during surgery. We hypothesized that AUS could be an alternative for diagnosis of patients with breast cancer as a non-invasive, less expensive, more accessible tool, than surgical pathological examination.

\section{Materials and Methods}

\section{Study design}

In this cross-sectional study, approved by Shiraz University of Medical Sciences (code:12190), patients who were referred to the clinic of the hospital from 28th June 2017 to 24th February 2018 were selected as the study population.

Among the patients who referred to the center during the study period, those who were diagnosed as having primary breast cancer based on physical examination, mammographic and sonographic results, indicative of suspicious tumor were referred for pathological examination, and in cases with positive results in the primary pathological examination, surgery was indicated, (mastectomy + sentinel lymph node biopsy based on their physician's order). At last 56 patients who did not have a locally-advanced disease or history of previous chemotherapy were included in the study. First, the patients were asked to read and sign the written informed consent form for their participation in this study. The patients' participation was voluntarily, and they were ensured that their information would be recorded by codes, and be used only for the purpose of this study.

All the included patients were scheduled for pre-operative axillary ultrasonography of the ipsilateral side of the involvement. Ultrasonography was performed in a supine or contralateral-side-down oblique position with Samsung ${ }^{\circledR}$ ultrasound (WS 80 ) unit by using a $5-10$ $\mathrm{MHz}$ linear transducer. The radiologist performing the AUS had 7 years experience in breast and axillary sonography, and recorded the results in the checklist, designed for this study. He started the scanning from the lowest part of the axilla and continued upward toward the axillary fossa to find all probable reactive or involved lymph nodes.

Cortical thickening of the lymph node (defined as thickness equal to or greater than the width of the fatty hilum), loss of fatty hilum, and morphologic grade of lymph nodes [divided into 6 grades (Pinheiro et al., 2014)], and presence of non-hilar blood flow were findings in favor of metastatic involvement of the lymph node. As loss of ovality of lymph nodes in AUS had a predictive value in diagnosis of lymph node involvement, the ratio of the largest to the smallest diameter of the lymph node was defined as lymph node ovality and shown as " $\mathrm{K}$ " in this study. So $1 / \mathrm{K}$ ratio indicated the loss of lymph node ovality as it gets closer to 1 .
Doppler sonography was also used to evaluate the vascular pattern of the nodes and distinguish between reactive and normal lymph nodes, by hilar vascularity, while malignant lymph nodes usually have a peripheral or mixed (hilar+ peripheral) vascularity (Ying et al., 2013). Finally, resistance index (RI), pulsatility index (PI), and systolic and diastolic velocity of the lymph node cortical vasculature were measured to find any probable relation between these factors and lymph node involvement.

Color Doppler study was done using Samsung ${ }^{\circledR}$ (WS 80) ultrasound system (L 3-8 MHZ linear array transducer). The setting for color Doppler sonography was optimized for each patient and wall filters, pulse repetition frequency, color gain, and focusing depth were adjusted accordingly. Then the patients underwent mastectomy + sentinel lymph node biopsy by the breast surgeon, who determined the sentinel node by dye, and took an intra-operative specimen of that lymph node for pathological examination. The specimens were kept in metal tissue disk, and they were transferred to freezer with $-20^{\circ} \mathrm{C}$ to $-30^{\circ} \mathrm{C}$ immediately, and sent to the hospital's laboratory in the shortest time possible, and then assessed and reported by a single pathologist. The initial results were reported to the surgeon during surgery, and if the results of frozen sections were positive, the surgeon would perform axillary lymph node dissection, and preserved the lymph nodes in formalin for 24 hours at room temperature. Then, they were transferred to the laboratory, and slides were taken from them for further examination. The researcher compared the reported results of pathology with AUS for evaluating the diagnostic accuracy of AUS.

\section{Statistical analysis}

The data recorded in the study checklist were input into MATLAB software (version 2014b) for comparison of the results and statistical analyses. The lymph node with the highest number of factors in favor of malignancy was selected for comparison with pathological results. We gave priority to the absence of fatty hilum and cortical thickening for the mentioned selection. Sensitivity, specificity, positive predictive value (PPV), and negative predictive value (NPV) were determined for the overall evaluation of AUS and histopathological results.

Predictive value of lymph node size (overall and cortical size of the lymph node), width to height ratio, morphological grade, vascular grade and pattern (mild, moderate and severe), the absence of fatty hilum, and the correlation between the number of involved lymph nodes in pathological reports with the probability of the diagnosis of metastasis were compared with pathological results, and reported by receiver-operating characteristic (ROC) curve and the area under curve (AUC) after exclusion of the patients with missing axillary lymph nodes in AUS (except in morphological grade, where missing axillary lymph nodes were considered as grade 0 ).

\section{Results}

A total of 56 patients with age of $47.58 \pm 12.28$ years (range 24-90) were involved in this study. Based on the pathological reports, lymph node involvement was 


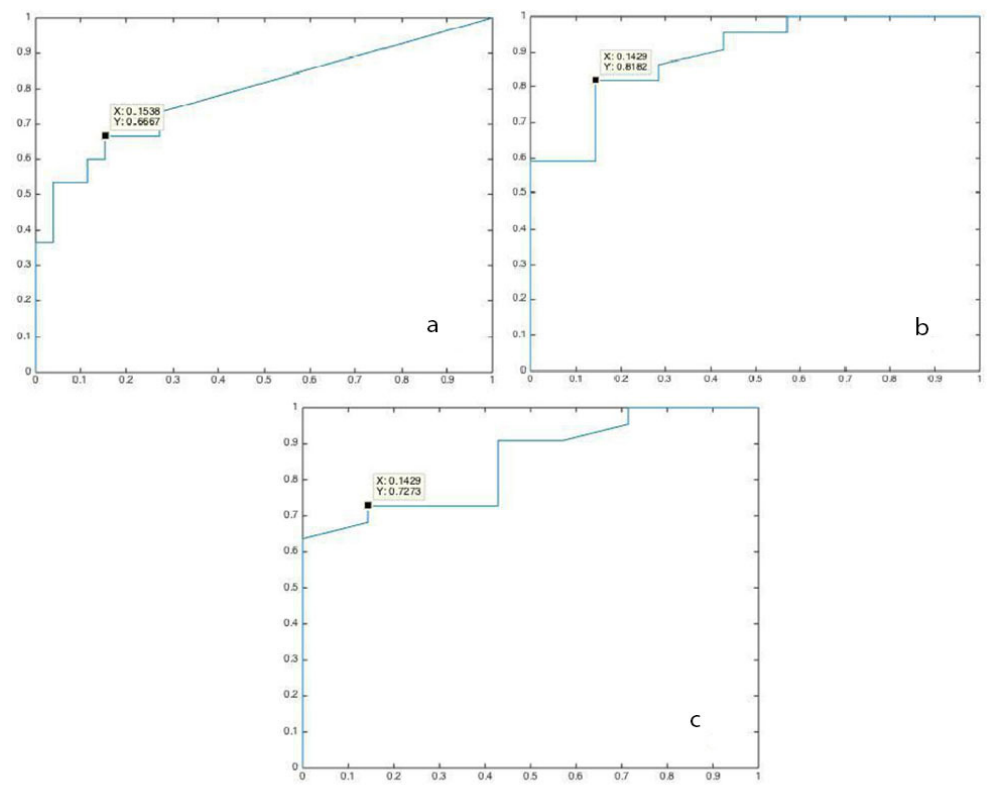

Figure 1. Receiver-operating Characteristic (ROC) Curve Calculation Containing X- axis for False Positive Rate (1-specificity) and Y-axis for True Positive Rate (sensitivity); a. Relation between lymph node size in sonography and pathology results $(n=56)$; b. Relation between lymph node cortex size in sonography and pathology results $(n=30)$; $c$. Relation between cortex size/lymph node diameter ratio in sonography and pathology results $(\mathrm{n}=30)$.

detected in 26 patients with benign and 30 with malignant disease, while based on ultrasonography results, 33 were diagnosed as having benign (58.9\%) and $23(41.1 \%)$ had malignant diseases, resulting in the sensitivity of $63.3 \%$, specificity of $84.6 \%$, PPV of $82.6 \%$, and NPV of $66.6 \%$ (Table 1).

Correlation between diagnostic features of lymph node involvement in AUS (explained in the method section) and pathology results revealed an AUC of $72.2 \%$ for lymph node size (95\% CI 59.2; 89.2) (Figure 1A). Assessing the correlation between the lymph node cortical thickness and probability of lymph node involvement in pathology showed a predictive accuracy of $89 \%$ (95\% CI 99; 77) with marked point of the cortical thickness at $3.7 \mathrm{~mm}$ after exclusion of patients with missing axillary lymph nodes in AUS (Figure 1B). Assessing the correlation between the ratio of cortex size/lymph node diameter (in its short axis) and pathological results showed an AUC of $85 \%(95 \%$ CI $95 ; 70)$ with marked point at 0.53 mm (Figure 1C).

Assessing the correlation between the lymph node sphericity in AUS and the pathological results showed increased risk of lymph node involvement as
$1 / \mathrm{K}$ approaches 1 (that shows the lymph node is more spherical) with AUC of $82 \%(95 \%$ CI $97 ; 64)$ and marked point at 0.41 (Figure 2).

The correlation between lymph node morphological grade, considering that missed lymph nodes were as

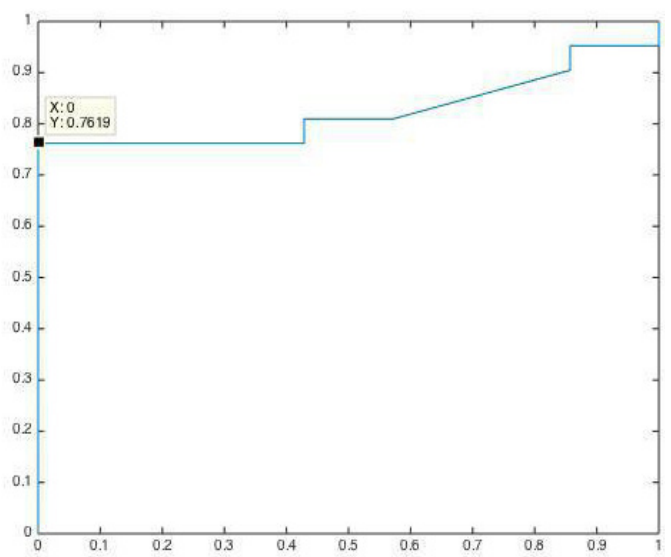

Figure 2. Receiver-operating Characteristic (ROC) Curve Calculation for the Relation between Lymph Node Sphericity in Sonography and Pathology Results Applied to Our Study Population $(n=30)$.

Table 1. Pathology * Sonography Findings Cross Tabulation

\begin{tabular}{|c|c|c|c|c|c|}
\hline & & & & & Total \\
\hline & & & Benign & Malignant & \\
\hline & benign & Count & 22 & 4 & 26 \\
\hline Pathology & & Percent within Pathology & 84.6 & 15.4 & 100.0 \\
\hline & & Count & 11 & 19 & 30 \\
\hline & malignant & Percent within Pathology & 36.7 & 63.3 & 100.0 \\
\hline Total & & Count & 33 & 23 & 56 \\
\hline & & Percent within Pathology & 58.9 & 41.1 & 100.0 \\
\hline
\end{tabular}



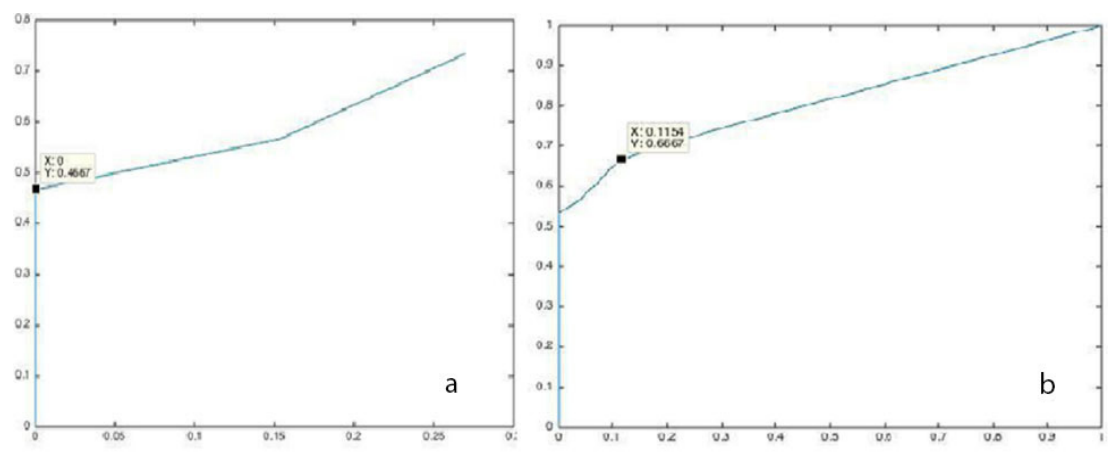

Figure 3. Receiver-operating Characteristic (ROC) Curve Calculation for a- relation between Lymph Node Morphologic Grade in Sonography and Pathology Results $(n=56)$; b- Relation between Shape of Lymph Node Hilum in Sonography and Pathology Results (n, 30).
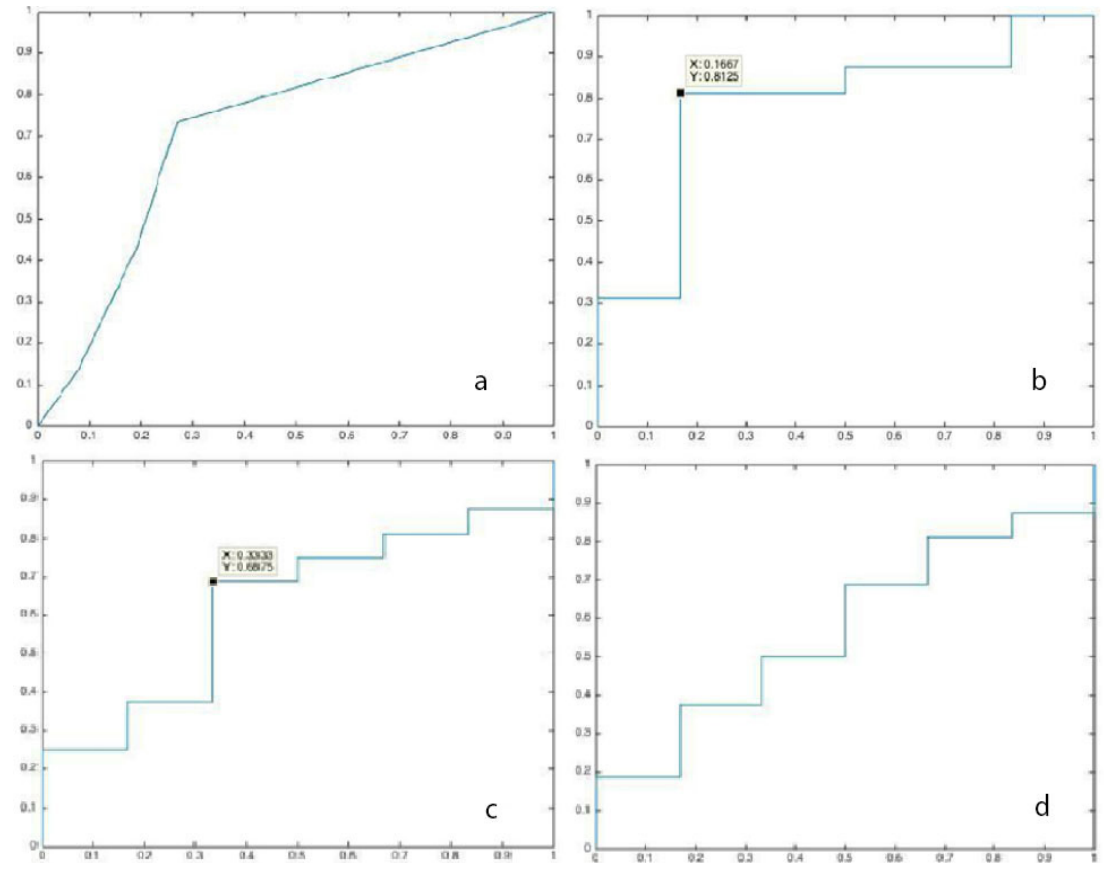

Figure 4. Receiver-operating Characteristic (ROC) Curve Calculation for a- Relation between Lymph Node Vascular Pattern in Sonography and Pathology Results $(n=30)$; b- Relation between systolic/diastolic flow in Doppler sonography and pathology $(n=30)$; c- Relation between Resistive index (RI) in Doppler sonography and pathology results ( $\mathrm{n}=30$ ); d- Receiver-operating characteristic (ROC) curve calculation for the relation between Pulsatility index (PI) in Doppler sonography and pathology results $(n=30)$.

grade 0 , revealed an AUC of $81 \%(95 \%$ CI $93 ; 70)$ with the marked point at grade 1 (Figure $3 \mathrm{~A})$. Assessing the predictive value of the lymph node hilum shape in sonography revealed an AUC of $78 \%(95 \%$ CI $87 ; 69)$ with the most specific point marked at the absent fatty hilum (Figure 3B).

The correlation between lymph node non-hilar blood flow revealed an AUC of 71\% (95\% CI 81; 63). The AUC of Resistive index (RI) and Pulsatility index (PI) indices were 62\% (95\% CI 81; 43) and 57\% (95\% CI 69; 36), respectively, and were not valuable predictive factors for diagnosis of axillary lymph node involvement. The AUC of systolic/diastolic flow (S/D) ratio was $78 \%$ (95\% CI 94; 59) (Figure 4 A-D).

Finally, studying the correlation between the diagnosis of lymph node involvement in AUS and number of involved lymph nodes in pathological findings revealed that the probability of diagnosis of lymph node involvement in AUS increased by more lymph nodes involved in pathological results. The sensitivity of AUS was $>93 \%$ in the involvement of two lymph nodes and

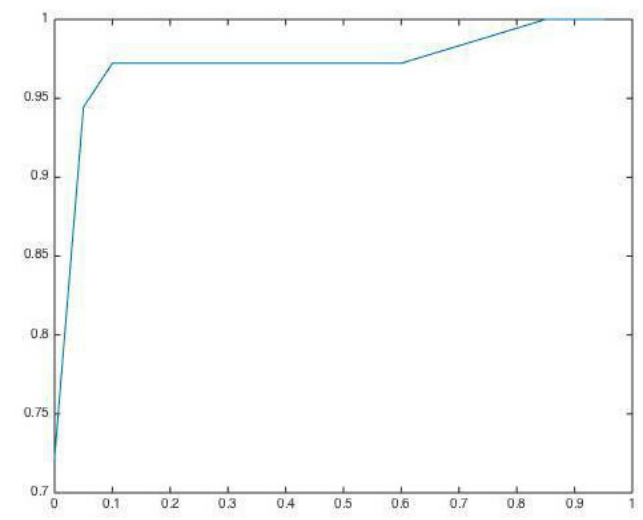

Figure 5. Receiver-operating Characteristic (ROC) Curve Calculation for Correlation between the Diagnosis of Lymph Node Involvement in Sonography and number of Involved Lymph Nodes in Pathology Results $(n=56)$. 
$>97 \%$ in the involvement of $\geq 3$ lymph nodes. The AUC of this figure is $92 \%(95 \%$ CI $94 ; 59)$ (Figure 5).

\section{Discussion}

The results of the present study, which aimed to determine the predictive value of AUS and lymph node characteristic in diagnosis of axillary lymph node involvement in patients with primary breast cancer, compared with the intra-operative pathological results, showed an overall sensitivity of $63.3 \%$, specificity of $84.6 \%$, PPV of $82.6 \%$, and NPV of $66.6 \%$ for AUS. These results indicate that AUS failed to diagnose all patients with lymph node involvement, as among 30 patients with malignancy according to pathological reports, only 19 patients were diagnosed appropriately. Nevertheless, further analyses showed that the sensitivity of AUS varied based on separated characteristics and increased to $>90 \%$, in some cases, as discussed further. Although our limitations in sampling lead to wide confidence intervals, the significance of the results shows that the mentioned factors role in the sonographic evaluation of axillary lymph nodes is not ignorable and further studies with greater sample size will strengthen the concluded results of this study.

Breast cancer staging (TNM) requires determination of lymph node involvement and as pathological examination is invasive, several researchers have been looking for non-invasive alternatives, including imaging modalities (Kumar et al., 2003). Hackney et al., (2013) reported the general sensitivity of $65 \%$ and specificity of $81 \%$ for AUS, which is close to the present study. But different studies have reported different sensitivity values for AUS, ranging from 23\% to 97\% (Bedrosian et al., 2003; Stachs et al., 2013). This variation among studies is generally based on the fact that the accuracy of ultrasonography depends highly on the operator's skills and experience and different accuracy of devices used that limits its standardization (Ohuchi et al., 2009). Therefore, in this study, AUS was performed by a single radiologist who had 7 years of experience in AUS and used an appropriate device to minimize this confounder.

In addition, as the results of the present study indicated, the number of lymph nodes involved had a direct association with the sensitivity of AUS and increased the sensitivity to $>93 \%$ for the involvement of two lymph nodes and $>97 \%$ for the involvement of $\geq 3$ lymph nodes. These results show that AUS can be a more accurate diagnostic tool in advanced stages, but not in early stages, which are consistent with the results of the study by Dihge et al., (2016) that determined the accuracy of AUS associated with metastatic size $(\mathrm{OR}=1.11)$, obesity $(\mathrm{OR}=2.46)$, and histological grade $(\mathrm{OR}=4.43)$. Therefore, not only patients' characteristics such as obesity, which limits the detection of lymph node involvement due to axillary fat mass (Shah et al., 2014), disease characteristics such as tumor type and lymph node size also play a role in the variable sensitivity (Rotim et al., 2017). As to the results of the present study, lymph node size had a predictive value of $72 \%$ and was not a definite factor in the distinction between reactive and involved lymph nodes, while disease stage, indicated by the number of lymph nodes involved, was associated with AUS sensitivity. These results are in line with what found in the study by Hackney et al., (2013) who reported that the sensitivity of AUS was related to the histological tumor type and decreased to $36 \%$ in lobular breast cancers.

Other lymph node characteristics were analyzed separately in the present study, which indicated that the cortical thickness and the hilum shape were the most reliable ultrasonography factors in diagnosis of axillary lymph node involvement in primary breast cancer. A lymph node cortex size of $3.7 \mathrm{~mm}$ had $81 \%$ sensitivity and $85 \%$ specificity and absent fatty hilum is the most specific factor for the diagnosis of lymph node involvement. Morphologic grade 1 had also a sensitivity of $66 \%$ and specificity of $88 \%$. These results are consistent with the results of Briton and colleagues, who indicated that AUS morphological characteristics, including absence of a hilum and a cortical thickness $>4 \mathrm{~mm}$ were associated with malignancy (Britton et al., 2009). In the study by Rotim et al., (2017), malignant lymph nodes had a higher maximal cortical thickness and the sensitivity of AUS exceeded $80 \%$ at cortical thickness of $5.1 \mathrm{~mm}$. In the present study, the ratio of lymph node cortex size to the whole width of the lymph node in its shortest axis had the highest predictive value (83\%), sensitivity of $72 \%$, and specificity of $85 \%$ at 0.53 , though it is only seen in high stages of the disease. These results are in line with the results of the study by Bedi and colleagues, who reported sensitivity, specificity, PPV, NPV, and overall accuracy of cortical shape in AUS at $77 \%, 80 \%, 36 \%, 96 \%$, and $80 \%$, respectively, for prediction of metastatic involvement of axillary nodes (Bedi et al., 2008). Therefore, the diagnostic accuracy of AUS is higher in some characteristics of the lymph node and combination of these morphological characteristics can be used for a more accurate diagnosis by AUS.

The highest specificity (100\%) in our study was related to $1 / \mathrm{K}$ ratio, indicating lymph node sphericity (measured as the ratio of the longest to the shortest diameter of the lymph node), and the risk of lymph node involvement increases as $1 / \mathrm{K}$ ratio approaches 1 (that shows the lymph node is more spherical) with predictive value of $82 \%$ at 0.41 , while the sensitivity was not high $(76 \%)$. As far as the authors are concerned, this is the first time that lymph node sphericity is reported as a sign, increasing the diagnostic value of AUS.

Lymph node Doppler sonography has been suggested by various studies for the assessment of the distribution of intranodal vascular pattern and resistance of lymph nodes (Ying et al., 2013) and has been reported to have high sensitivity and specificity for differentiating metastatic and reactive nodes (Gupta et al., 2011). In the present study, the results showed a positive correlation between lymph node non-hilar blood flow and lymph node involvement. It is noticeable that this factor could not distinguish involved and reactive lymph nodes precisely. The ratio of the systolic to diastolic blood flow also showed a positive correlation with pathological results, though it had a large confidence interval; while the other two vascular factors (RI and PI) showed no correlation with pathological 
results. There seems to be much controversy about the diagnostic value of RI and PI in the literature; some suggest higher RI and PI in metastatic than reactive nodes (Cui et al., 2013), while others suggest lower or similar RI and PI in metastatic than benign nodes (Moritz et al., 2000). Accordingly, these indices are considered with limited application in routine clinical practice.

The results of the present study could be limited by various aspects. First, any diagnostic errors of the radiologist performing the AUS or the ultrasound device could have affected the results. Second, we could not compare the lymph node characteristics with pathological results, as the aspects recorded were dissimilar in some cases. Therefore, it is suggested to compare the results of preoperative AUS with intra-operative AUS for better assessment and comparison.

In conclusion, as our results revealed, AUS is a valuable but not a definite tool for determination of axillary lymph involvement in primary breast cancer and has a high sensitivity at high disease stages with macro-metastases. Therefore, it is suggested to use AUS for general assessment, but not for definite diagnosis. It seems like sensitivity and specificity of the AUS could be raised by adding new parameters like lymph node sphericity to the diagnostic criteria. The operator dependency of ultrasonography is undeniably influences the results, and the similarity between involved and reactive lymph nodes is another challenging part of the diagnosis. For better evaluation of this issue, further studies with a larger population and intra-operative AUS are recommended for comparison of the characteristics of each lymph node detected with pathological evaluation of the same lymph node.

\section{Funding Statement}

The present study was financially supported as M.D thesis by Shiraz University of Medical Sciences (code: 12190).

\section{Acknowledgements}

The authors of the present study sincerely thank Dr. $\mathrm{S}$. Tahmasbi for her kind cooperation in case preparation.

\section{References}

Abe H, Schmidt RA, Kulkarni K, et al (2009). Axillary lymph nodes suspicious for breast cancer metastasis: sampling with US-guided 14-gauge core-needle biopsy-clinical experience in 100 patients. Radiology, 250, 41-9.

Bedi DG, Krishnamurthy R, Krishnamurthy S, et al (2008). Cortical morphologic features of axillary lymph nodes as a predictor of metastasis in breast cancer: in vitro sonographic study. AJR Am J Roentgenol, 191, 646-52.

Bedrosian I, Bedi D, Kuerer HM, et al (2003). Impact of clinicopathological factors on sensitivity of axillary ultrasonography in the detection of axillary nodal metastases in patients with breast cancer. Ann Surg Oncol, 10, 1025-30.

Berg WA, Blume JD, Cormack JB, et al (2008). Combined screening with ultrasound and mammography vs mammography alone in women at elevated risk of breast cancer. JAMA, 299, 2151-63.
Britton PD, Goud A, Godward S, et al (2009). Use of ultrasound-guided axillary node core biopsy in staging of early breast cancer. Eur Radiol, 19, 561-9.

Cui XW, Jenssen C, Saftoiu A, et al (2013). New ultrasound techniques for lymph node evaluation. World J Gastroenterol, 19, 4850 .

da Costa Vieira RA, Biller G, Uemura G, et al (2017). Breast cancer screening in developing countries. Clinics, $\mathbf{7 2}$, 244-53.

DeSantis C, Ma J, Bryan L, et al (2014). Breast cancer statistics, 2013. CA Cancer J Clin, 64, 52-62.

Deurloo E, Tanis P, Gilhuijs K, et al (2003). Reduction in the number of sentinel lymph node procedures by preoperative ultrasonography of the axilla in breast cancer. Eur J Cancer, 39, 1068-73.

Diepstraten SC, Sever AR, Buckens CF, et al (2014). Value of preoperative ultrasound-guided axillary lymph node biopsy for preventing completion axillary lymph node dissection in breast cancer: a systematic review and meta-analysis. Ann Surg Oncol, 21, 51-9.

Dihge L, Grabau DA, Rasmussen RW, et al (2016). The accuracy of preoperative axillary nodal staging in primary breast cancer by ultrasound is modified by nodal metastatic load and tumor biology. Acta Oncol, 55, 976-82.

Ertan K, Linsler C, Di Liberto A, et al (2013). Axillary ultrasound for breast cancer staging: an attempt to identify clinical/ histopathological factors impacting diagnostic performance. Breast Cancer Basic Clin Res, 7, S11215.

Ghoncheh M, Pournamdar Z, Salehiniya H (2016). Incidence and mortality and epidemiology of breast cancer in the world. Asian Pac J Cancer Prev, 17, 43-6.

Gupta A, Rahman K, Shahid M, et al (2011). Sonographic assessment of cervical lymphadenopathy: Role of high-resolution and color Doppler imaging. Head Neck, 33, 297-302.

Hackney L, Williams S, Bajwa S, et al (2013). Influence of tumor histology on preoperative staging accuracy of breast metastases to the axilla. Breast $J, \mathbf{1 9}, 49-55$.

Houssami N, Ciatto S, Turner RM, et al (2011). Preoperative ultrasound-guided needle biopsy of axillary nodes in invasive breast cancer: meta-analysis of its accuracy and utility in staging the axilla. Ann Surg, 254, 243-51.

Jazayeri SB, Saadat S, Ramezani R, et al (2015). Incidence of primary breast cancer in Iran: Ten-year national cancer registry data report. Cancer Epidemiol, 39, 519-27.

Jung J, Park H, Park J, et al (2010). Accuracy of preoperative ultrasound and ultrasound-guided fine needle aspiration cytology for axillary staging in breast cancer. ANZ J Surg, 80, 271-5.

Kumar R, Jana S, Heiba SI, et al (2003). Retrospective analysis of sentinel node localization in multifocal, multicentric, palpable, or nonpalpable breast cancer. J Nucl Med, 44, 7-10.

Leong SP, Shen ZZ, Liu TJ, et al (2010). Is breast cancer the same disease in Asian and Western countries?. World J Surg, 34, 2308-24.

Moritz JD, Ludwig A, Oestmann JW (2000). Contrast-enhanced color Doppler sonography for evaluation of enlarged cervical lymph nodes in head and neck tumors. AJR Am J Roentgenol, 174, 1279-84.

Mousavi SM, Montazeri A, Mohagheghi MA, et al (2007). Breast cancer in Iran: an epidemiological review. Breast $J$, 13, 383-91.

Ngoma T (2006). World Health Organization cancer priorities in developing countries. Ann Oncol, 17, 9-14.

Ohuchi N, Suzuki A, Sakurai Y, et al (2009). Current status and problems of breast cancer screening. JMAJ, 52, 45-9.

Pan HB (2016). The role of breast ultrasound in early cancer 
detection. J Med Ultrason, 24, 138-41.

Petropoulou T, Kapoula A, Mastoraki A, et al (2017). Imprint cytology versus frozen section analysis for intraoperative assessment of sentinel lymph node in breast cancer. Breast Cancer (Dove Med Press), 9, 325.

Pinheiro DJPdC, Elias S, Nazário ACP (2014). Axillary lymph nodes in breast cancer patients: sonographic evaluation. Radiol Bras, 47, 240-4.

Rotim T, Kristek B, Turk T, et al (2017). Measurable and unmeasurable features of ultrasound lymph node images in detection of malignant infiltration. Acta Clin Croat, 56, 415-24.

Schwartz TL, Margenthaler JA (2017). Axillary ultrasound before neoadjuvant chemotherapy for breast cancer: Don't discount the benefits yet!. Ann Surg Oncol, 24, 618-20.

Shah AR, Glazebrook KN, Boughey JC, et al (2014). Does BMI affect the accuracy of preoperative axillary ultrasound in breast cancer patients?. Ann Surg Oncol, 21, 3278-83.

Shah TA, Guraya SS (2017). Breast cancer screening programs: Review of merits, demerits, and recent recommendations practiced across the world. J Microsc Ultrast, 5, 59-69.

Siegel RL, Miller KD, Jemal A (2018). Cancer statistics, 2018. CA cancer J Clin, 68, 7-30.

Stachs A, Göde K, Hartmann S, et al (2013). Accuracy of axillary ultrasound in preoperative nodal staging of breast cancer-size of metastases as limiting factor. SpringerPlus, 2, 350.

Ying M, Bhatia K, Lee Y, et al (2013). Review of ultrasonography of malignant neck nodes: greyscale, Doppler, contrast enhancement and elastography. Cancer Imaging, 13, 658.

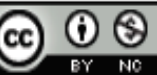

This work is licensed under a Creative Commons AttributionNon Commercial 4.0 International License. 